\title{
Research
}

\section{Integrating online communities and social networks with computerised treatment for insomnia:}

a qualitative study

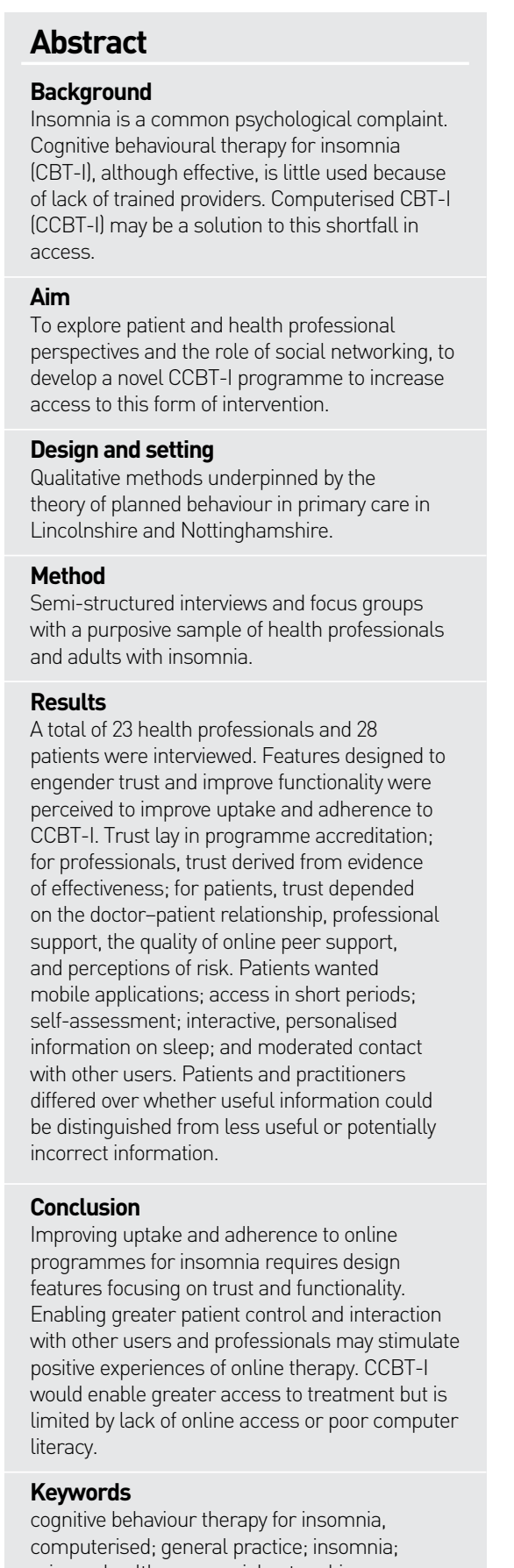

\section{NTRODUCTION}

Insomnia, the most commonly reported psychological complaint in Britain,' is linked healthcare use and costs.

Hypnotic drug therapy is widely used despite concerns about its safety and limited evidence of its effectiveness. Cognitive behavioural therapy for insomnia CBT 1) has been show to be as effective term for persistent insomia $8-12$ and for comorbid insomnia associated with anxiety.

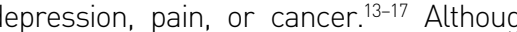
programmes such as Improving Access to Psychological Therapies (IAPT) 18 now provide better access to non-pharmacological therapies for conditions such as anxiety and depression, CBT-l is not widely use Computerised cognitivent. herapy (CCBT) may be a solution to this access shortfall. CCBT is now a recommended treatment for anxiety and depression, and access to such programmes 5 increasing in the UK. 1,22 There is also growing evidence for the effectiveness of
CCBT for insomnia (CCBT-I) $23-26$ despite CLBT for insomnila (CCBT-I), ${ }^{2-26}$ despite
its limited availability. Furthermore, little is known about potential users' expectations or experiences of such programmes. Existing programmes, while innovative, do not reflect the full range of people's experience of computer technologies,
which may include engagement with online J Middlemass, MA, MPhil, research assistant; Health and Social Care, University y f Lincoln, Lincoln KCavanagh, DPhil, DClinPsych, senior lecturer in
clinical psychology, School of Psychology, University of Sussex, Brighton. CLinehan, PhD, lecturer, School Morgan, PhD, professsor of gerontology; School of

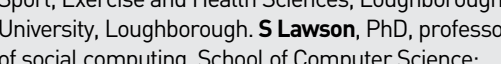
of social computing, School of Computer Sciences
University of Lincoln, Lincoln. AN Sirivardena. University of Lincoln, Lincoln. AN Sirivardena,
MMedSCi, PhD, FRC GPP. professor of primary \& pre- communities and social networks that may increase participation and completion rates for CCBT-I.

While previous research has explored patient and professional attitudes to standard CCBT programmes for common mental health problems, ${ }^{20-30}$ no published study has focused specifically on potential user attitudes towards integrating CCBT-1 attitudes are an important predictor of treatment intentions in this context 31,32 The aim of this study was to investigate patient and professional perspectives, including attitudes, expectations, and beliefs towards any online healthcare programmes that they had used to inform development of a novel CCBT-I platform. More specifically, the researchers aimed to explore and how they would negotiate information disclosure to health professionals or fellow insomniacs; identification of factors that would encourage referral, take-up, and adherence; and the effectiveness of a uniquely integrated CCBT-I and social networking application

METHOD

A qualitative design was used to collect sampled professionals and patients. Both semi-structured interviews and focus group methods were used to maximise the breadth and depth of perspectives. Participants

\section{Social Care, University of Lincoln, Lincoln.}

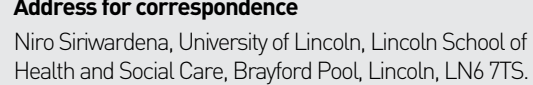
Health and Social Care, Brayford Pool, E-mail: nsiriwardenalalincoln.ac.uk OBritish Journal of General Practice This is the tull-length article ppublished online
26 Nov 2012 of of an abridged version published in print. Citie this article as:
10.3399/bigp 226559321
How this fits in

Cognitive behavioural therapy for insomnia
ICBT-Il has been demonstrated to bo

(C)

that adults would be willing to be referre

refer to an online computerised CBT-

moderated and it had design features that increased trust and functionality. Results platform for CCBT for insomnia and other

health conditions.

were recruited from Lincolnshire and patients treated by their GP for insomnia, and potential service users with sleep problems (but for ease of terminology both are referred to as patients in this articlel. A range of health professionals working with patients altected by sleep problems were recruited through posters in public places and family practice waiting rooms, while health professionals were recruited from direct mailing to practitioners or via clinical leaders. A description of the health professionals and patients interviewed is Thatle from the aulhors i

The pallent sample was recruited on the basis of their having sleep problems Additionally, patients who had and had not used CBT and/or CCBT were recruited, to understand more broadly views about the potential of CCBT-I and reduce bias about the focus on a particular (C)CBT programme. Both patients currently seeking treatmen and those not cuntenly seekng treatment groups may have been different, the study sought to maximise patient variation and the responses generated. Health professiona participants were purposively recruited on the basis of their profession and the length of time they had practised. In addition, those who had and had not facilitated access to CBT and CCBT for palients were recruited recruitment process was stopped when participants were not providing any new avenues of enquiry, that is data saturation was achieved.

Interview schedule pic guide (Appendix attitudes, expectations, and beliefs including behavioural intentions and any mediating factors relating to online health care that might facilitate or create barriers to uptake
and adherence to CCBT-I. Patients were and adherence to CCBT-I. Patients were
asked about their sleep problems, how they had tried to solve them, and where they had found useful advice lif indeed they hadl. Past or current behaviour around using the internet, electronic devices, or applications for health-related matters was explored, to improve understanding of patients' and practitioners' subjective norms, attitudes towards CCBT-I, and beliefs as
to whether they would access CCBT-I with to whether they would access iCBT-I with
a social networking element in the future. Thus, social networking practices were investigated in relation to what, how, and when, users communicated online and any preferences and barriers that were current treatment for patients presenting with insomnia were explored, as well as their views about both health information

The theory of planned behaviour was approach because the theory has been shown to inform design features that will lessen attrition and heighten adherence in CCBT. ${ }^{33}$ The theory of planned behaviour proposes that intention to perform a behaviour is an immediate precursor to performing that behaviour and is related norms lthey are expected to engage in the behaviourl and perceived behavioural control (the person is able to engage in the behaviourr. ${ }^{34}$ Qualitative methods were used to understand participants' beliefs about the outcome of performing the behaviour, about what other people wanted them to do, and about their abilities and the avaliability aread with their health care in relation to CCBT-I and social networking in Intenview data were managed using NVivo 8. Thematic analysis was used, and codes and themes were developed iteratively as data were collected. ${ }^{3}$ This allowed the researchers to explore new avenues of enquiry during the fieldwork. This process generating initial descriptive codes, and searching for underlying themes, reflecting deeper notions and conceptualisations. . $^{3,37}$ All the codes constructed were data driven (inductive), within a broader framework relative to theory of planned behaviour. The framework consisted of beliefs about and 
any mediating factors that may help design ways that pon suited to the contemporary The process involved three of the authors developing initial codes, two of the authors continuing to code data to generate new codes and potentiat themes, and three ogether in an iterative fashion, to generate the final themes and quotes that best illustrated these. Divergent views were actively sought and the different perspectives of the multidisciplinary research team considered during analysis.

RESULTS

Twenty-eight patients and 23 health January and July 2011 at health premises, he university, or patients' homes. Thes included: 17 individual and three focus group interviews with patients and eight interviews and three focus groups with professionals. Two meta-themes emerged from the data: trust and functionality (Box 1). Trust and confidence in the programme. Functionality referred to the range and quality of functions that improved the usability and usefulness of the programme for patients. Trust for both health professionals and patients lay in accreditation of programmes; or professionals, trust also derived from the evidence of efrecliveness, whereas for patient, relationship, ongoing professiona support and feedback, and the quality of peer support, including perceptions of security and risk.

Box 1. Meta-themes, themes,

Trust

Trust tin the programme
o Evidence of effectiveness D Accredited and non-commercia
DProfessional advocacy

- Trust in the patient-professional relationship 0 Part of the package of care
o Feedback to professional carers Direct access to online treatment

- Trust in online peer support
o Sharing information ol Information security and stranger danger - User homophily
- Asynchronous preferable to synchronous - Lurking versus sharing
0 Moderation of posts

\section{Functionality}

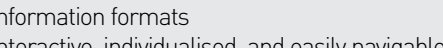
- Interactive
Timing

GP22: I think it's useful to have evidence that it does actually make a difference. Also GP17: 'Yes if I thoe ort som or risk.

useful and t thought that there's reasona evidence to back it up, then I would feel happier to recommend it and perhaps mor confident in suggesting it to people.' IGP locus group - GP22: GP principal, male; GP17: GP principal, male)

GPs acknowledged that CCBT-I could be a useful treatment option but felt tha tor mental health problems before referral. Some had referred for CBT or CCBT, with viable experiences. Very few patients with 政 of what CBT-I was, although some had condition CBT for alher ment health

My sister who suffers a little bit from anxiety as well ... I said "Oh you want to ask your doctor about CBT", and I was trying to explain it to her, and the one thing I found that I couldn't be tangible, I couldn't say "Oh well it gives you this and it gives you that and I sounded like I was talking about some dunno, leftist type of alternative therapy
which CBT isn't really at all it sounded like I was asking her to sort of burn the oils. y'know. ' (patient 26: male aged 35 years)

Additionally, participants felt that CCBT-I should be delivered through accredited, n-commercial organisations:

I would want to know a bit more about it. I would want to know about its provenance. this, is it a commercial organisation is it a professional health organisation, is it an educational institution, where's it come from?... I'm not sure I would have faith that my doctor had actually checked out because hey might just be passing it on ... How know it?'

If it was accredited by a university or medical good start.' (GP focus group - GP22: GP principal, male

If there was a direct link from a GP practice, oo that means that we've already vetted it that NICE [National Institute for Health and Clinical Excellence guidancel agreed to IGP focus group - GP22: GP principal, male) to be one of the big differences.' '(CMHT 08: sychological wellbeing practitioner, male) Professional support for specific programmes was also enthanced or leedback from patients:

Most people haven't liked [x] website because it's very intense It just takes onger, it's intellectually actually very very challenging, so I think a lot of people just give up. They get fed up of the intellectual effort they have to put into it. ... Just random survey people just hated [x].' (G focus group - GP17: GP principal, male

Trust in the patient-professional eel they were being treated dismissively by being referred to CCBT-I if they trusted the referring GP and if CCBT-I was part of package of ongoing care:

think if it was a programme like on prescription, go away ' a like you to do this fine because you know that it's continuing care. It's not like off you go and don't come back.' (patient focus group - patient 22. female aged 43 years)

Patients generally supported the notion of their progress within CCBT-I being fed back to a health professional, particularly those

Some patients wanted to select which were raised in relation to continuity of care patients did not regularly see the same ealthcare professional:

I think one of the problems with GPs thes days is usually you don't see the same GP. we're on a carousel so you get whoever comes next and for me to see my GP to lalk about my withdrawal plan, I'm going to have to book 2 weeks in advance. That's the really of modern healli care. (patient tocus group - patient 18: male aged 50 years)

Health professionals were open to consent, provided that it did not require too much additional work, because they felt hat identifying and monitoring patterns of esponse to treatment might be clinically helpful. Direct access to CCBT-I was an mpottant consideration for those who did for insomia:
If you don't see it [insomnia] as a problem, and you haven't gone searching for like thing .... I tend not to go to the doctors for anything.' (patient 21 :

frust in online peer support. Some patients were happy to share their experiences relating to insomnia and communicate with others online. They felt it would be isolation to know that there were other people with the same condition. Others wanted to communicate online but remain completelyanonymous or use a pseudonym:

'Oh it would be nice to have a pseudo-name! ... It's like being in the corner with a bag on

Fear of not knowing who else was online was a concern for many. Additionally, giving out personal information, which might end up in the wrong hands, or leaving a digital of having used the sitel which might be accessed by others, were concerns:

It think I'd be a bit reluctant if I didn't know them. They'd have to be friends... but I don't know about strangers, and I don't think [husband] would be happy with me talking to strangers in the night or whatever. Ipatient ale aged 45

Im very wary of the internet, we leave digital footprints wherever we go and you never know what's going to come back and haunt you and I think the more that you are in a professional working environment the you put online rou be careful about what certain parameters' got to keep it within aged 56 years)

Among those who were happy to engage in online chat with others, there was a prefrence to discuss issues only with per particular condition, for example bereavement or a mental health problem:

If your sleep was related to say trauma, or your sleep was related to pain, or bipolar both a general sleep body where you could participate but also go down to the subgroups and maybe get some specific advice. Because / do think you listen to your own group.' (patient focus group - patien
18: male aged 50 years) 
Patients considered asynchronous rather than synchronous communication to be safer, that is posting a note, commenting on a forum, or adding to a thread and ratings others' postings, rather than engaging
online communication in real time:

But rather than have it almost real time because that might creat an is necessary really. So it would be nice to be able to read somebody's comments that they might have left several hours before. (patient 26: male aged 35 years)

Some participants preferred to be lurkers, looking at others" online ideas or questions. IA 'lurker' is a person who reads online discussions on a forum/ thread or other interactive systems, but rarely or never actively participates or contributes.l Reasons given for this were lack of time, and red

Normally if you do a search you'll find that someone's had the same issue as you, you ind out what they've done to rectify thos issues and take that information away from I'm not being selfish because it's already out there... Somebody's already had the issue so there's no point me putting that

Despite a perceived risk that lay ideas or solutions might be inaccurate, most patients thought they would be able to evaluate online advice appropriately.
professionals were less convinced.

You'd just weigh it up and decide whether anybody is going to post that has not been the press or wherever. It's certainly going to be something really unusual isn't it? (patient focus group - patient 09: female aged 52 years)

It could be open to all sorts of things being said and suggested and then patients are is actually recommended and what is't. (NP18: nurse practitioner, female)

Moderating online CCBT-I was felt to be important. Some suggested that al users could be moderators, while others suggested that users could progress to become moderalors. A system with users was also suggested, although participants practice.

\section{Functionality}

Participants suggested a range of function usability and usefulness of the prove the for patients.

Information needs. Some CCB programmes were perceived to be more user friendly than others, in terms of layout, readability, and complexity:

'[x] is [a] much easier, user-friendly website. It's easier to read, the fonts are bigger, the hing that patients are looking for. 'IGP focus group - GP22: male GP principal)

The study data did not suggest any sexor age-related differences in responses to potential use of CCBT-I, whereas limite access to computers due to financia disabilities were considered barriers:

because if you look at some people that do have sleep problems ... it may be that nobody has asked them about their literacy skills, so to actually say go and read this, go and look online, they may not be able to, or they might not be able to read the Other people might have visual problems. Some people might have hearing problems. so it's very much [an] individual thing. (NP18: nurse practitioner, female)

Nonetheless, patients highlighted the need to have accurate, research-based intion that was regularly updated

'As long as it's got the right information and it's up to date, I don't like it when you go in and click on something and it's 1999 and it's a press release, well we're in 2011 now so. . page! li's got to be fresh all the time you (patient 36, male aged 51 years)

Patients wanted to know what the normal sleep pattern was, how theirs compared with the norm, and whether it merited medical attention:

I need to know that my sleep pattern was doing more harm than good ... because I think ils not normal, but whether its it's outside of $i t$ I wormally or whelts what point the medical profession would consider a sleep pattern as something to do something
56 years)

Completing a sleep diary was seen by patients as a way of identifying a pattern to lifestyle causes for which they welcomed personalised feedback. Professionals felt sleep hygiene was valuable and that their patients either did not know about or did not implement this advice, whereas patients felt that they already knew about and had tried sleep hygiene. ISleep hygiene is a se that patients are advised on to help the overcome their insomnia.)

Most of them already have a mindset, as soon as you start talking about sleep hygiene being the way to go, they switch of hat may be a prejudice of mine but that's my observation

I've seen a psychologist and gone through sleep hygiene and things but it doesn't really work... ve tried all the sleep hygiene things. the same time and all that, have a milky drink or bath. I've done all that.' Ipatien tocus group - patient 02: female aged 45 years

A number of participants wanted information on the causes of insomnia and the drugs available to treat it (prescribed or over the counter). Onine lectures and professional advice were also suggested.

Information formats. Some patients favoured an audio format with audio-books, preferred a more visual approach, reflecting differences in learning styles:

Well I always think, because I don't believe in one particular style dominating an individual's learning capacily... in terms of stimulation and recall 1 just think a bit of everything for me personally.' Ipatient 40

Visual formats using photos or animations, games, puzzles, or humorous content were suggested to engage and encourage interactive learning:

I'd quite like e-learning to be interactive. so you're doing things along the way rather puzzles or something, have a picture of bedroom with various things in it and say Which of these things are not conducive to you having a good night's sleep, and so you being turned up full blast and/or do you want extra curtains or whatever, so things And you get feedback that says maybe wive found five things there and there were 10 there $\ldots$ or whatever' 'pharmacist focus group - CP 14: male community pharmacist)

Seeing videos of others with sleep sharing their stories was considered helpful:

Actually it would good to have a video because you could then see the person, than just reading ... because you feel it worked for so and so and soil could work for 29. as well. (patient focus group - patient male aged 23 years

Text was the least popular format. Only one patient said they preferred text, whereas many others felt it would be a barrier to reading from Some participants did not whe concerned about problems with reading skills. Participants suggested that text, if used, should be in shor blocks and easy to

I can read a page, and then by the time I've read the other page / ve forgotten what the first page said so have to go back again." (patient 31: male aged 38 years)

Participants varied in preferences for the level of information detail. Some wanted in 'bite sized', easy-to-read chunks with headlines, bullet points, or summaries. Others wanted the opportunity to access more detail if desired.

hreractive, Individualised, and easily input to be Patients wanted information input to be interactive, individualised, and of how this might work. They wanted to document a sleep diary and lifestyle factors that generated a personalised plan advice and recommendations:

'Possibly, maybe a custom-built plan for the individual .... inputting individual feedback or advice, tips a ne ridutidual reedback or advice, tips, and products you 
asked a few things, like what have you had to eat or whatever, how much do you drink, caffeine or Whatever, and how much do you smoke and then maybe, a timetable, whe to get up when to go to bed, how long you should leave before going to bed after you ve caffeine or smoke.' 'patient 15. male aged 20 years)

Patients wanted flexibility to select learning that they felt might be most relevant to them, using a search facility or requently asked questions. Several patients wanted to access ideas and tips onlise could not sleep:

If I had sleep app on it Imobile phone], when can't sleep / can just go on it and it might just help with sleep or something. I might be just scrolling and think, somebody has just sald this helps so IIl try it." (patient focus

Links signposting patients to other sites were suggested to reduce overcin the site and enable users to access mor detailed information:

Timing. Most users were prepared to access CBT-I for short periods but not necessarily every day.

the was not an issue; they would spend as long as was needed to get what they needed out of the package, with

Most people would struggle to concentrate on anylhing for more than 7 minutes don't they? That's what IVITV channell reckons. something like that.' (GP focus group GP22: male GP principal

Again that's difficult because the time that you spend will be in relation to the amoun that you need to know and the amount of information that's avallable. You would give it the time that you fell it needed, so if it got out of it I would spend whatever time took.' (patient 21: female aged 56 years)

\section{DISCUSSION}

Two main themes were identified: trus and functionality. Features designed to increase trust in CCBT-I and improve programme functionality were perceived uptake and adherence. Patients were more likely to accept a credible evidence-based programme for insomnia when referred by a trusted professional as part of ongoing perceived to provide muth users, while perceived to provide mutual support, gave information security Patients wanted mobile applications, access in short periods, selfassessment, and a personalised, interactive approach; they also wanted contact with others to be moderated or overseen

\section{Strengths and limitation}

The strengths of this study were the triangulation of data /confirmation of findings from different groups of participants), and divergent case analysis from a wide range of patients and health professionals. This was a multidisciplinary study involving academics from general practice, nursing, sleep science, psychology, social science, and human-computer interactions, affording a from one area of the UK which may not reflect experiences elsewhere, but the authors are confident that their methods generated conceptual generalisability. ${ }^{39}$

\section{Comparison with existing literature}

Professionals and patients in this study wanted CCBT-I to be accredited, nonfriendly. Previous studies have also found health information to be more credible if it is accredited, 60.41 and/or endorsed by government or a reputable organisation. ${ }^{42}$ Users have also been shown to be wary of commercial interests and concerned about advertisements or promotional materials. Individuals wanted CCBT- 1 to be control over what information they accessed, when, and how. They preferred information in short blocks with visual and audio features rather than just text. Language, clarity. tone, and comprehensibility of information are key presentational considerations but breadth, depth, accuracy, and scientific quality have also been shown to be infortant for credibility of online health using a professional design and quality the opportunity to offer (or be offered) treatment other than a sleep hyglene leaflet or hypnotic drugs. Referral for CCBT-1 was considered to be more acceptable relationships were deemed good and part of a care pathway. Previous research has

found that referral and monitoring by health professionals tends to increase trust, Whereas lack of trust in health professionals to greater dissatisfaction with services. ${ }^{40}$ However, those who did not wis wanted to be ab to acess CCBT-I directly. Interaction with other online CCBT-I users via a social networking interface was felt to provide mutual support, particularly if there were perceived similarities between users (so called homophily') and if interactions were moderated. In contrast, there wer concerns about privacy or latent 'threats' to personal safety. In one study, "Ipoor] preference for online social interaction: This, in turn, predicted negative outcomes associated with problematic internet use. ${ }^{49}$ concerns that were not voiced by participants of the present study. There are notable similarities and differences between online health illness) and social networking sites Sites such as Facebook tend to foster pre-existing social relationships ${ }^{50}$ where users are more likely to interact regularly with a smal number of online friends while passively following the status of a larger number of acquaintances." Online health communities,

Funding

EPSRC Healthcare Partnerships EP/ 1000615/1: Exploring social Networks to Augment Cognitive behavioural Therapy

Enct

Leicestershire, Northamptonshire and Rutland Research Ethics Committee governance approvals were gained from both Nottinghamshire and Lincolnshire Primary Care Trust and Mental Health Trusts.

Provenance

Freely submitted; externally peer reviewed. Competing interests

Kate Cavanagh is a consultant to Ultrasis plc who market Beating the Blues. The have declared no competing

\section{Acknowledgements}

We would like to thank the Mental Health Research Network and Primary recruit participants, and the patients and professionals who generously participated.

Discuss this article

Discuss this article

Contribute and read comments about http://www.rcgp.org.uk/bjgp-discuss
Although patients in the present study felt they would be able to distinguish accurate from were more doubtful about this

Users in the present study preferred greater control over interactions through responses. Both asynchronous and realtime (synchronous) online communication have advantages and disadvantages. While some users dislike slower feedback, others prefer the convenience of asynchronous communication, as it can be difficult to talk to others because of different time an opportunity to read and respond to a posting and it may be difficult to find a particular individual online at any one time. ${ }^{54}$

Implications for practice and research

The rapidy increasing familiarity and use of socialnetworking provides an unprecedented opportunity for health interventions to delivered interventions with peer-to-peer support are showing positive effects on health outcomes..$^{55}$ The findings of this study provide detailed evidence on design features that users suggest may improve uptake and adherence to online health interventions for insomnia and other mental health complaints. Further research is needed to attributes is associated with increased use, greater adherence, and consequently improved health outcomes.

- Improving uptake and adherence to online programmes for insomnia requires design features focusing on trust and functionality. Enabling greater patient control and interaction with other users axperiences of online therapy CCBT twould enable greater access to treatment but is limited by lack of online access or poor computer literacy.
Kalth support groups in
required to read others' issues, have been perceived to have greater and therefore increase oppor

.

a




\section{REFERENCES}

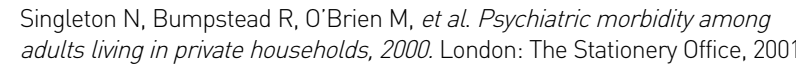

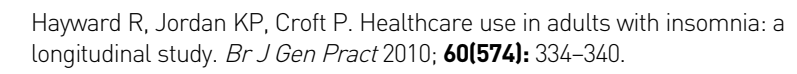

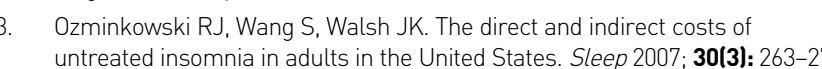

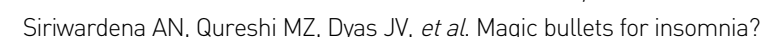

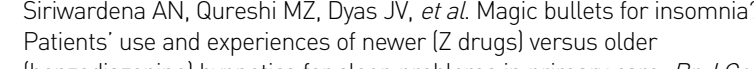

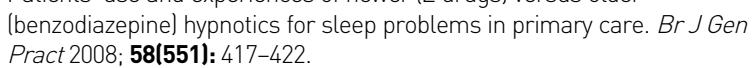
Glass Lancto k

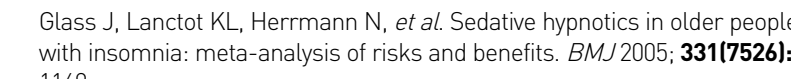

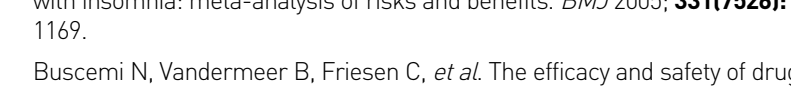

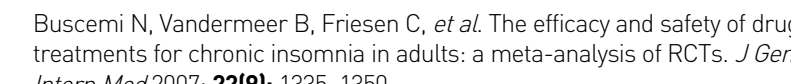

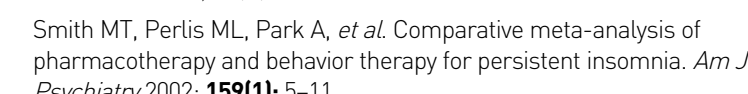

8. Imvin MR, Cole JC, Nicassio PM. Comparative meta-analysis of behavioral interventions for insomnia and their efficacy in middle-aged adults and
older adults 55 y years of age. Health Psychol 2006; 25(1): $3-14$. Montgomery P. Dennis J. Cognitiviv behavioural interventions for sleee
problems in adults aged $60+$. Cochrane Database Syst Rev 2003; (1):
CDo03161.

10. Morin CM, Hauri PJ, Espie CA, et al. Nonpharmacologic treatment of chronic insomnia. An
$1134-1156$.

11. Morin CM, Bootzin RR, Buysse DJ, et al. Psychological and behavioral
treatment of insomnia:update of the recent evidence (1998-2004). Sleep 2006; treatment of insomnia
29(11): $1398-1414$.

12. Morgan $\mathrm{K}$, Dixon $\mathrm{S}$, Mathers $\mathrm{N}$, et al. Psychological treatment for insomnia in the regulation of
$8(8)$ : iii-68.

13. Smith MT, Huang MI, Manber R. Cognitive behavior therapy for chronic insomnia occurring within the context
Clin Psychol Rev 2005; 2555): 559-592.

14. Manber R, Edinger JD, Gress JL, et al. Cognitive behavioral therapy for insomnia enhances depression outcome in patients with comorbid major

15. Vitiello MV, Rybarczzk B, Von KM, Stepanski EJ. Cognitive behavioral therapy

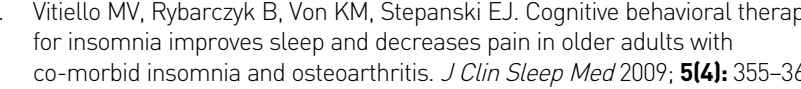
16. Jungquist CR, O'Brien C, Matteson-Rusby $\mathrm{S}$, et al. The efficacy of cognitive-
behavioral therapy for insomnia in patients with chronic pain. Sleep Med behavoral therapy for

17. Espie CA, Fleming L, Cassidy J, et al. Randomized controlled clinical effectiveness trial of cognitive behavior therapy compared with treatment 26(28): $4651-4658$.

18. Department of Heath. Improving Access to P Psychological Therapies (IAPT):

19. Mckinstry B, Wilson P, Espie C. Non-pharmacological management of
chronic i insomnia in primary care. Br G Gen Pract 2008; 58[547): 79-80,

20. Neylan TC. Time to disseminate cognitive behavioral treatment of insomnia-
comment on Efficacy of brief behavioral treatment for chronic insomnia in comment on 'Eficacy of brief behavioral treatment for
older adults'. Arch Intern Med 2011; 171(10): 895-896.

21. National Institute for Heath and Clinical Excellence. Computerised cognitive

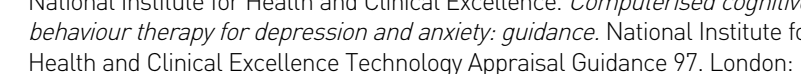
Health and
NICE, 2006 ,

22. Department of Health. Improving Access to Psychological Therapies. IAPT

23. Strom L, Pettersson R, Andersson G. Internet-based treatment for insomnia.
a controlled evaluation. $J$ Consult $C$ Clin PSychol 2004; 72(1): 113-120.

24. Vincent N, Lewycky S. Logging on for better sleep: RCT of the effectiveness of
25. Ritterband $L M$, Thorndike FP, Gonder-Frederick $L A$, et al. Efficacy of an
Internet-based behavioral intervention for adults with insomnia. Arch $G$ en Psychiatry 2009: 6677): 692-698.

26. Espie CA, Kyle SD, Williams C, et al. A randomized, placebo-controlled trial of online cognitive behavivoral therapy for chronic insomnia disorder delivered
via an automated media-rich web application. Sleep 2012; 35166: $769-781$.

27. Thorndike FP, Saylor DK, Bailey ET, et al. Development and perceived utility and impact,

28. Kaltenthaler E, Sutcliffe P, Parry G, et al. The acceptability to patients of computerized cognitive behaviour therapy for depression: a systematic
review Psychol Med 2008: 38111: 1521

29. Williams C., Whitifield G. Writtinen and computer-based self-help treatments for

Stallard P, Richardson T, Velleman S. Clinicia sus :

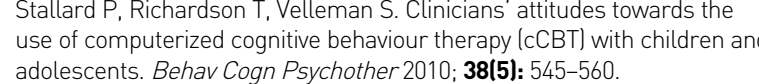

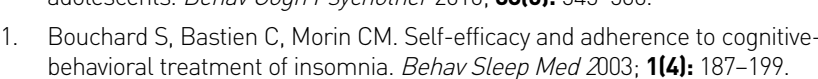

32. Hebert EA, Vincent N, Lewycky S, Walsh K. Attrition and adherence in the
online treatment of chronic insomnia. Behar Sleep Med 2010; 833: $141-150$.

3. Webb $T L$, Joseph J, Yardey L, Michie S. Using the internet to promote head theoretical basis, use of behavior change techniaues, and mode of delivery on efficacy. J Med Internet Res 2010; 12(1): e4.

34. Ajzen I. From intentions to actions: a theory of planned behavior. In: Kuhl
J, Beckmann J, eds. Action control. from cognition to behavior. Berlin, NY: 1985; 11-39.

35. Barbour RS, Kitzinger I. Developing focus group research: poltitics, theory

36. Boyatzis RE. Transforming qualitative information: thematic analysis and

code development. Thousand Oaks: Sage Publications, 198

37. Braun V. Clarke V. Using thematic analysis in psychology. Qual Res Psych
$2006 ;$; : 77-101.

38. Coyle D, Doherty G, Matthews M, Sharry J. Computers in talk-based mental
health interventions. Interact Comput 2007; 19(4): $545-562$.

39. Mays $N$, Pope C. Qualitative research in health care,
qualitative ressearch. $B M J$ 2000; 32017226): $50-52$.

40. Eysenbach G, Kohler $\mathrm{C}$. Hoy

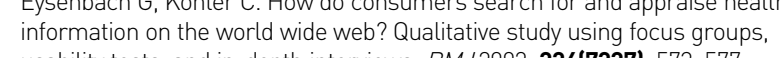
usabilityty tests, and in-depth interviews. BMJ 2002; 324.73337: $5733-577$.

41. Freeman KS, Spyridakis JH. An examination of factors that affect the
credibility of online health information. Tech Commun 2004; $51: 239-263$. 42. Metzger MJ. Making sense of credibility on the Web: models for evaluating Online intormation and recommendations
Inform Sci Tech 2007; $58(13):$ : 2078-2091.

43. $Y$ Y $Y$. Correlates of consumer trust in online health information: findings from
the health information national trends survey. $J$ Health Commun 2011; 1611

44. Ye $Y$. A path analysis on correlates of consumer trust in online health Intoremation: evidence trom the health intormations
Health Commun 2010; 15(Supp 3): 200-215.

45. Sirivardena AN, Apekey T, Tilling M, etal General practitioners' preferences 'tor managagng insomnia and opportunities for reducing hyponotic prescribing.

46. Dyas JV, Apekey TA, Tilling M, et al. Patients' and clinicians' experiences of consultations in primary care for sleep problems
group study. Br $\mathcal{S}$ Gen Pract 2010; 601574$): 180-200$

47. Thom DH. Physician behaviors that predict patient trust. J Fam Pract 2001:
50(4): $323-328$.

48. Thom DH, Kravitz RL, Bell RA, et al. Patient trust in the physician
relationship to patient requests. Fam Pract 2002; 1955: 476-483.

49. Caplan SE. Preference for online social interaction: a theory of problematic
Internet use and psychosocial wellbeing. Commun Res 2003; 30: $625-648$.

50. Newman MW, Lauterbach D, Munson SA, et all. I't's not that I don't
have problems, I' $m$ just not putting them on Facebook: challenges a opportunities in using online social networks for health. CSCW Proceedings of the ACM 2011 Conference on Computer Supported Cooperative Work,
51. Economist. com. Social networks - primates on Facebook: even online,
the neocortex is the limit. The Economist 26 February 2009. http://mmm The neocortex is the limit. The Economist 26 February 2009. http:///wm

52. Winzelberg A. The analysis of an electronic support group for individuals with
eating disorders. Comput Hum Behar 2012 2 ;3: 393-407.

53. White M, Dorman SM. Receiving social support online: implications for health
education. Health EduC Res 2001; 1666: $1993-707$
54. Wright K. Perceptions of on-line support providers: an examination of within on-line support groups. Commun Q2000; $48: 44-59$.

55. Bender $\mathrm{JL}$, Radhakrishnan A, Diorio C, et al. Can pain be managed through the Internet? A sys 


\section{Appendix 1. Topic guide}

- What are health professionals and patients experiences with cognitive behavioural therapy (CBT)/ computerised CBT (CCBT)/CCBT for insomnia (CCBT-I) and why it may, or may not have worked in the past

- What would encourage GPs land other health professionals) to refer patients for CCBT-I and would patients be willing for that referral?

- What do GPs/other health professionals and patients think about communicating progress from the completed CCBT-I package to their GP or other nominated health professional?

- What would patients and health professionals like to see in the CCBT-I package and how would they like it presented?

- How long would patients be willing to spend on the CCBT-I package per day?

- What do patients think about communicating including through an online social network with others

suffering from sleep problems as part of the CCBT-I package?

- What ethical processes and safeguards need to be considered and in place? 